\title{
UN gender network workshop III: report
}

Conference or Workshop Item

Published Version

Freedman, R., O'Donoghue, A. and Doherty, S. (2018) UN gender network workshop III: report. In: UN Gender Network Workshop III, 14th November 2017, Durham University. doi: https://doi.org/10.2139/ssrn.3106897 Available at https://centaur.reading.ac.uk/98984/

It is advisable to refer to the publisher's version if you intend to cite from the work. See Guidance on citing.

To link to this article DOI: http://dx.doi.org/10.2139/ssrn.3106897

Publisher: United Nations Gender Network

All outputs in CentAUR are protected by Intellectual Property Rights law, including copyright law. Copyright and IPR is retained by the creators or other copyright holders. Terms and conditions for use of this material are defined in the End User Agreement.

\section{www.reading.ac.uk/centaur}

\section{CentAUR}

Central Archive at the University of Reading

Reading's research outputs online 


\section{UN Gender}

Network

Workshop III:

Report

Durham University, November 14th 2017 Prof Rosa

Freedman, Dr Aoife O’Donoghue, Sophie Doherty 


\section{Contents}

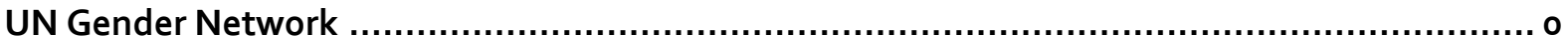

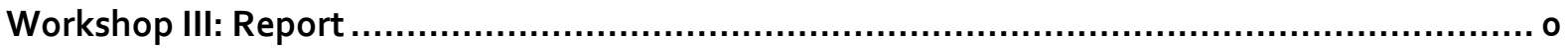

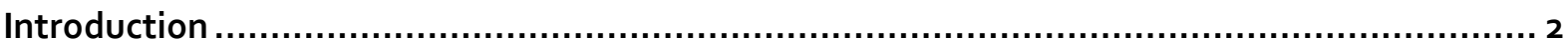

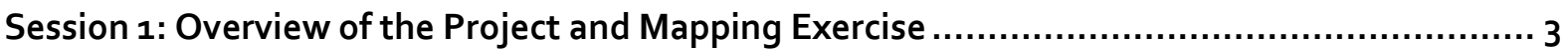

Session 2: United for Gender Parity and the UN Secretary General's Proposals.....................8

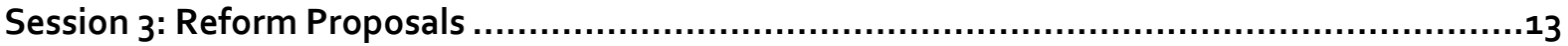




\section{Introduction}

The following is a record of the third UN Gender Network Meeting hosted by Durham University on November 14th 2017. The AHRC funded UN Gender Network brings academics, civil society, member states and those who work at the United Nations together in a spirit of conversation and collaboration. Our core focus is on the UN Secretariat and UN Agencies and the working lives of women within those organisations. A feminist outlook is taken in examining the continued gender inequality within the United Nations and we consider an intersectional and inclusive definition of women to be central to our understanding of equality. The Network also aims to set forth potential policy and legal change to the UN Secretariat and Agencies to bring about gender equality. 


\section{Session 1: Overview of the Project and Mapping Exercise}

Introductory remarks focused on what the Network had achieved thus far and the publication of both the UN Secretary General's Report on the Improvement in the Status of Women in the United Nations System Report in July 2017 and the New System Wide Strategy on Gender Parity and the new United for Gender Parity website. A brief overview of the Women Peace and Security Event hosted at the London School of Economics with Navi Pillay, Jane Connors, Rose Park and Aoife O'Donoghue was also given.

As quite a few people in attendance were new to the Network Sophie Doherty gave an overview of the first two workshops. This included detail of what was discussed, including definitional issues at the first workshop as well as an overview of the state presentations at the second workshop.

In returning to some of the key themes of earlier workshops several definitional issues were re-examined. This included several key themes. First, how to ensure that our feminist methodology was evident in how we describe ourselves, second the need for an inclusive definition of women that would not exclude intersectional questions as well incorporating the lived experience of Transgender women into our deliberations and third the need to assert that geographic diversity was central in understanding the Sustainable Development Goals. (SDGs).

In examining these issues, the conservative outlook of the United Nations as an organisation was a key consideration in any strategic attempts to engage with the institution. It was further discussed that recent changes to policies around marriage recognition as well as the re-launch of goals toward gender parity perhaps suggested some other possibilities for engagement.

In embracing an avowedly feminist approach to the project considerations of the failed policies of the past within the United Nations Secretariat and Agencies and, as such, the need 
to account from existing lessons around cultural change through accountability, leadership, unconscious bias awareness, deadlines and prioritisation as well as the layering of intersectional issues amongst staff was critical. Exploiting resources from amongst the Network would enable challenges to be met in developing policy proposals.

Consideration of access was also discussed. How to gain support for potential policy reforms, how to ensure women's voices and calls for change were heard - particularly from the Global South - were considered essential. A further consideration was the difference between gender parity and gender equality and how we as a group out to reflect that difference.

A discussion of leadership and the role of women in 'traditional' leadership positions within the Organisation unfolded, this included the role women play in human rights sections and agencies within the United Nations and the need for their inclusion in 'non-traditional' areas. In doing so there was discussion of how member states, can lead by putting women forward but also what lessons can be learned from elsewhere in moving women into non-traditional parts of the Organisation. However, some notes of caution were also put forward. This included the UN's tendency to utilise the achievements of women member state delegates as their own, including on the new UN Gender Parity website, the use of non-Secretariat and Agency moment as indicators of improvements for women in the Organisation including CEDAW as well as the reliance of 'great' moments for the actions of women to be noted as opposed to the everyday contributions that women make to the Organisation.

\section{Mapping}

Roslyn Park and Molly Whitley - from The Advocates for Human Rights - gave an overview of the initial outcomes of the Mapping Exercise undertaken of the UN Secretariat and Agencies Human Resource Policies and Statistics. Over the previous months The Advocates for Human Rights had organised and facilitated over 20 interns and associates to examine in detail the UN's extensive human rights policies. Each intern filled out a template form in order to allow the Network to gain a better picture of the United Nations policies. This was deemed necessary following discussions at the first workshop where the plethora of policies and statistics as well as the difficulties in locating all of these were highlighted. Roslyn and 
Molly presented an initial report with a more detailed report to following once a full coding of the documents is completed.

Rose Park introduced the work completed over Summer 2017 and both Rose and Molly noted the difficulties that the interns had in garnering the data. This included the variance within the Secretariat as to the public nature or otherwise of documentation, the multiplicity of sites where information was available, the rate at which documentation would disappear from the UN website or that web links would regularly break, the extent to which some policies appeared to be cut and paste from one place to another. Statistics were also inconsistent with some referencing recent dates such as 2016 but others dating back to 2010 . This made collecting the data difficult but also meant that it was problematic to claim any completeness regarding the conclusions. The presentation also focused on the professional staff however it was noted that when you include General Services that the statistics often look much better with regard to gender parity. The number of women in General Services - which include largely administrative tasks -is a key consideration that also needs to be noted.

The types of policies the associates looked at included:

- Harassment/Bullying/Exploitation

- Childcare

- Breastfeeding

- Parental Leave

- Maternity/Paternity/Adoption Leave

- Surrogacy Policy

- Whistle-blower/Anti-retaliation Policy

- Discrimination Policy

- Intern Policy

- Early Career Policy

- Marriage Recognition

- Gender and Diversity Policy

- Recruitment of Underrepresented Groups

- Progression and Recruitment 
- Special Measures

- Monitoring Process for Implementation of Gender Targets

- Gender Impact Statement Policy

- Senior Staff Recruited Internally/Externally

- Training and Development

- Talent Identification Process

- Mentoring/Coaching/Networking

- Exit Interview/Process

Molly presented details of bodies all of which fell under the Secretariat and as such required to use the policies as set out in the Human Resources Handbook. Molly noted the use of jargon in the handbook but also the lack of definitions in the text that can be vague arguing that someone would need guidance to understand the implications of the text. Molly discussed how the language was often non-obligatory with terms such as 'shall consider' rather than imperatives. Molly also noted that the HR Handbook is the minimum required and that each Department is encouraged to go beyond it to respond to its employee needs.

Molly then noted some good practices from the UN. For instance, the UN Development Programme (UNDP) which has been the model for other agencies in some of its practices. For example, UNDP extended the breastfeeding policy of the Secretariat. The Secretariat's policy applied to those expressing milk or nursing with infants under the age of one year. UNDP has expanded that to include employees with children up to two years of age in line with the World Health Organisation's recommendations. Further UNDP has women-only candidate pools for Bureaus that have fallen below $45 \%$ representation and men are targeted for general services positions. UNDP also has a Leadership Development Pathways program started in 2016 with two courses. First, to increase capacity of managers to create gender inclusive workspace, and second, to focus on women and leadership, addressing the challenges women face in and on the way to leadership, and actions all can take to help women overcome those challenges, though it is difficult to tell if these are mandatory 
courses. The UNDP Gender Equality Seal Initiative is a corporate certification process recognising good performance in delivering transformative results for gender equality.

Several other agencies also had positive policies, for instance UNICEF has a career counselling program that focuses on the needs of each employee and can take into account gender-specific needs or issues. The program also has a gender balanced selection of mentors. UNICEF designed a Men in Leadership course to raise general awareness about sensitivity to gender issues. UNICEF instituted an exit survey focusing on the effectiveness of UNICEF's policies regarding families and balancing work and personal obligations and workplace culture. Interestingly in 2006, UNICEF brought in an outside consultant to study gender parity at the senior levels of management. The results were quite negative, finding, among other things, that though the policies in place were good in theory, many women were hesitant to take advantage of them because it was seen as career threatening. It was also found that the emphasis on long hours and output made staff members feel like UNICEF encouraged them to treat each other as "units of work rather than human beings with real lives to lead and hold together." Daily microaggressions along with the feeling of an old boys' network made female employees feel othered and undervalued. This study was done over ten years ago and was made public. It is unclear whether it has been repeated and what the impact of changes following the consultant's report actually were. A point of concern that was raised was whether UNICEF continued to work with the consultant when such a negative report was issued, as often Organisations move to a different consultancy or stop the process altogether.

Another example of good practice that Molly outlined was the International Maritime Organization's Integration of Women in the Maritime Sector programme that supports the inclusion of more female Maritimes amongst the IMO's member states. The idea is that these women can subsequently be recruited into service at the IMO. At the UN Conference on Trade and Development (UNCTAD) knowledge and experience on gender is listed as a competency requirement for hiring. In addition, demonstrating knowledge and expertise on gender would then enhance candidates' chances to be recruited by UNCTAD. The Department of Peacekeeping Operations has a specific page on their website titled 
"Attracting Senior Women" on which they declare that they "are looking for senior qualified women with proven leadership skills, integrity, and commitment to the ideals of the UN Charter to create a 'talent pipeline' of Directors in UN Peacekeeping and Special Political Missions." But Molly raised questions regarding whether its implementation has succeeded as there is no public data.

However, Molly also emphasised that these were limited examples of good practice and while commended there was great variance across the Secretariat between policies. The lack of statistical or other information also meant it was difficult to assess the success or otherwise of specific policies. In making recommendations to other bodies it was difficult to ascertain whether these policies ought to be replicated. Molly also emphasised that the difficulty the group faced in finding these policies would mean that UN staff or those applying to work at the UN would face similar difficulties. While some policies may be password protected, for staff in the field or for those who were considering working at the UN the difficulties in seeing policies ought not to be underestimated. Molly asked why exactly such policies and statistics need to be confidential. Molly argued that the UN ought to be the model for employers around the world and that being comparatively better than states or Organisations was insufficient. The UN will succeed in its leadership if it can point to its own good practice.

\section{Session 2: United for Gender Parity and the UN Secretary General's Proposals}

The workshop then moved to the new Report from the Secretary General as well as the new United for Gender Parity Strategy. This was introduced by Aoife O'Donoghue. Aoife began her presentation by quoting the Secretary General António Guterres; 
Gender parity at the United Nations is an urgent need and a personal priority. It's a moral duty and an operational necessity. The meaningful inclusion of women in decision-making increases effectiveness and productivity, brings new perspectives and solutions to the table, unlocks greater resources, and strengthens efforts across all the three pillars of our work. ${ }^{1}$

Aoife welcomed the new emphasis that the Secretary General had brought to gender equality and parity. It was noted by Paula Schreifer the importance of the recognition that gender equalities increase effectiveness and productivity.

Aoife then discussed the United for Gender Parity website and in particular the toolkit. Aoife noted that many of the elements were campaigns such a HeforShe which was an outwarded looking campaign as well as the gender milestones often being state focused treaties or state based events. Aoife noted that the history of women in the Organisation was often that of women state delegates rather than women working within the United Nations infrastructure. Aoife also noted the partial history that was presented which did not include older General Assembly resolutions on gender parity which began in the 1986 but also the lack of information on for instance the failure to meet Boutros Boutros Ghali's target of 50/50 by the $50^{\text {th }}$ anniversary of the creation of the United Nations. There are some positives on the website including the new strategy itself and links to the Talent Pipeline. The lack of statistical information was noted but also the further plans for the website which will include more information on the Organisation.

In the strategy itself Aoife noted several elements including that it aims to balance accountability with incentives, it recognises the different starting points and challenges across the Organisation and that it aims to provide positive tools to empower and encourage staff and managers. That the document stated that, '[t]he goal, however, is not just about hitting the numbers. It is about modernizing the organization and shifting its institutional

\footnotetext{
${ }^{1}$ System Wide Strategy on Gender Parity 2017 available at https://www.un.int/sites/www.un.int/files/Permanent\%20Missions/delegate/1700102b_gender_strategy_report_13_sept_2017.pdf Prof Rosa Freedman, Prof Aoife O'Donoghue, Sophie Doherty http://blogs.reading.ac.uk/united-nations-gender-network/
} $\mathrm{AH} / \mathrm{P006027/1}$ 
culture so that the United Nations can access and capitalize on its full potential' was also welcomed.

The inclusion of disaggregated targets was welcomed including three target dates addressing international staff, FS, P-1 to USG levels, on Permanent/Continuing and Fixedterm appointments: 2021,2026 and 2028. To ensure parity by these dates and guidance on annual targets for each entity/level combination based on the gap to parity albeit the language used here was of recommendation rather than obligation.

Aoife noted that the document stated that the Organization as a whole is now 17 years behind on its due date for parity however this excludes the earlier targets set by Boutros Ghali and while the phrasing has changed over the years the intended substantive outcomes were the same. Aoife noted that in acknowledging that it recognised that it had an issue much earlier but has failed to achieve this may give more impetus for change rather than ignoring its past failures albeit the Report clearly intends to set a positive tone about the potential for change which is welcome. Aoife also noted some caution around the focus on doing this in accordance with Article 101 and the criteria of merit, and should not weaken the quality of staffing welcoming the Report statement that 'the assumption that the recruitment and promotion of women would somehow lessen standards rather than raise them is refuted by evidence' but that more needed to be done regarding Article 101. Aoife also welcomed the clear statement that 'gender parity should not be conflated with the totality of the UN's efforts on gender equality.' Aoife then outlined some of the policies included in the document including:

- Include in Senior Managers' Compacts specific, timebound, and entity specific targets for reaching parity at all levels, based on the methodology described in "Target Setting"

- Request non-secretariat Executive heads to sign similar Compacts with calculated targets voluntarily to be monitored and reported on to the CEB Secretariat

- Monitor compliance and hold senior managers accountable for meeting targets, including through taking corrective actions 
- The achievement of gender parity will require considerable effort and accountability should also be matched with public recognition of those who perform and provide positive leadership as well as real incentives for institutional transformation

- Responsible to Head of Entity to reach the calculated time-bound, entity and level specific targets within their departments/offices/missions

- If targets are not met by December 2019, the department/office/mission's selection authority will be revoked for the under-represented grade level(s), and the central HR office will make all selection decisions for the under-represented grade level(s) for one year

- If following the resumption of selection authority, targets are not met for the following year, this will be reviewed by the Secretary-General directly for decision

- Good practices guidelines for an enabling and inclusive organizational culture are developed by CEB HR Network, OHRM and UN Women and shared with each entity to incorporate into existing policies, monitoring and implementation

- Every entity to ensure it has in place a strong legal framework on sexual harassment and that staff are aware of support in place and actions to take

- To ensure that efforts in one area are not undermined by efforts in another, any organizational change should entail a gender assessment impact study - this is particularly important with current reform processes

- Replace maternity/paternity/adoption leave policies with one parental leave policy where parental leave (maternity, paternity, adoption and surrogacy) is equal in length, provided that specific pre- and post-delivery needs of the birth parent are covered additionally (including insurance and leave) in line with WHO's recommendation of 6 months for breastfeeding/ bonding with child

- Allow for additional 6 months of parental leave for staff in non-family duty stations

- Entities to ensure on-site creche availability for children or alternatively a percentage reimbursement of costs for creche where there is no facility

- Ensure that all policies and measures are applied equally to opposite and same-sex spouses/partners including their recognition as beneficiaries of staff members by the UNJSPF irrespective of the staff member's nationality

Prof Rosa Freedman, Prof Aoife O'Donoghue, Sophie Doherty $\mathrm{AH} / \mathrm{P} 006027 / 1$ 
- Revisit all Host Country Agreements to see which can include spousal employment visas or where it would be possible to reopen discussions on the issue

Aoife noted however the difference between mandatory and recommendations in the language and the import of deliberating on how this ought to be measured and implemented across the Organisation. Aoife welcomed this new initiative and the attention and focus that the new Secretary General had brought to the issue but on the basis that there was room for improvement.

The workshop then moved into group discussions focusing specifically on the Appendix 1 of the System-Wide Strategy on Gender Parity. One of the issues with Appendix 1 first mentioned was its partial reflection of the document as a whole, the choice of what to include or exclude from this Appendix would be significant as departments and agencies may use the Appendix as a short-hand model. The tense of the language was also noted, that is was generally in passive voice and abstract, so who would be responsible for its implementation and development was unclear. Further who would monitor the success or otherwise of both the policies and individual agencies and department. The grouping of elements on recruitment under flexible working arrangements also seemed problematic. The lack of clear definitions of much of what was included was also noted and the implications that would have for implementation discussed. An example of this appeared to be the interchangeable nature of sexual harassment/abuse as opposed to workplace bullying which are different and needed to be explicitly outlined as such.

A clear issue was raised regarding the idea of women as exceptions to the minimum standards. That a certain type of women was understood as working at the Organisation and that they needed exceptional assistance rather than the paradigm shifting to as to establish that women were the norm within the organisation and certainly not the only ones that might consider flexible working arrangements or requiring family friendly policies. The focus on breastfeeding was also remarked upon as it did not appear to account for the fact that children require care beyond infancy. While the value of women to the workplace is clearly acknowledged in the Report this needed to be utilised in the minimum standards that the 
Organisation expects to demonstrate that women were not an exception to be dealt with but also different that women made a variety of choices.

The resources necessary to implement the standards outlined, for instance the reliance on technology - the timelines for implementation and the use and quality of statistical data to collate and measure the success of these standards also seemed largely absent.

Several omissions from the Annex were also noted - while acknowledging that some were in the body of the Report itself - this included childcare and caring responsibilities more generally, the possibility of intimate partner violence both of and by UN staff members, the effect of absences on women's career prospects and how this might be ameliorated, the potential for abuse relationships between staff members and the impact this has on retention of women. The absence of discussion of 'family postings' and the need for mobility was also a central issue as well as the absence of discussion of gender based health concerns that can make participation in field activities more difficult if they continue to go unconsidered. Issues regarding diversity, disability, LGBTQI also were necessary to ensure that the Organisation welcomed all women. Whether the recommendations would lead to a cultural shift that is needed in the Organisation was discussed. The need to shift the culture so as to give women a sense of belonging in the Organisation was deemed necessary.

A discussion of the nature of 50/50 targets, about how sensitive that figure needed to be and the possibilities of a plus/minus needed to be teased out. A discussion also preceded on the role of various bodies in the implementation of this report including UN Women, the Focal Points for Women and the CEB and the use of this across several entities and how this could impact on its implementation.

A final point that was raised was who would hold the UN to account should these minimum standards and the other recommendations contained in the strategy not succeed in achieving gender parity or creating substantive gender equality.

\section{Session 3: Reform Proposals}


In the final session of the workshop Aoife O'Donoghue presented the group with alternative reform proposals. These proposals are intended to stand alongside the System-Wide Strategy for Gender Parity and act as a gold standard for the Organisation. The significant differences between the Strategy and the Draft Document included the direct assertion of a legal mandate for gender equality based on the Pre-amble, Article 1.3, Article 8 and Article 101. The proposal to have the UN undergo an evaluation as to whether it would be the requirements of the ILO's Gender Standards, CEDAW and Sustainable Development Goal 5, a comprehensive set of minimum standards that would be obligatory, the re-invigoration of the Focal Point for Women, the making of temporary special measures permanent, and the introduction of a qualitative survey of all staff. The policy also recommends that the Organisation looks at its own historical narrative and the artwork within the facilities.

Discussions that followed included the need for specific definitions for gender equality and parity, the need to make some of the language less academic, whether it would be possible for the Network to undertake the assessment of the Organisation under the standards outlined ourselves, what the consequences for failure ought to be, can this include loss of authority or increments, the need for detail in places for instance requiring managers to have remedial plan if gender targets go into regression, not just reporting. Consider the funding of the re-invigorated Focal Points for Women as this was a large part of its lack of success, to separate recruitment from promotion and retention. To define success and what that would mean for the Organisation.

On a broader point of strategy, it was discussed whether a long policy proposal was a good idea or whether we should confine ourselves to three-four items to optimise success. And the need to consider who we wish to talk to at this point. Those in attendance were also asked to submit further comments to Aoife O'Donoghue and Rosa Freedman. 